\title{
Magnetic field computation and simulation of the coil systems using Comsol software
}

\author{
Ivan Zatonov*, Pavel Baranov, and Andrey Kolomeycev \\ National Research Tomsk Polytechnic University, 634050 Tomsk, Russia
}

\begin{abstract}
The article considers the calibration system computation with the inhomogeneity of magnetic field less than $0.1 \%$. Method of calculation is described. The numerical simulation using finite-element analyze was made for such system as: Helmholtz coils, improved Helmholtz coils, four and six coils system. Authors made the calculation of the magnetic field homogeneity toward axial direction. Based on procure results, authors analyzed magnetic field homogeneity and compared different types of coil systems.
\end{abstract}

\section{Introduction}

The measuring of magnetic flux density plays the key role in such brunches of since and technology like: task of orientation, navigation, stabilization, flaw detection, nondestructive testing, magnetic tomographic measurement, shielding action for quantum computer, etc.

Magnetometer gains currency for magnetic field measuring [1]. The stormy developing of robotic engineering complexes of surface-mounted, underwater and space system appropriation requires magnetometers evolution that will provide opportunity for weak magnetic field measuring. The theme of magnetic field measuring was reviewed by following scientists $[2,3]$.

Permanent field source of magnetic flux density is used for magnetometers calibration and graduation. The inaccuracy of magnetic field source must be three times less than the inaccuracy of the calibrated sensor. For instance, for the measuring device with inaccuracy near of $1 \%$, it is necessary to provide homogeneity magnetic field with inaccuracy 0.1 $0.3 \%$.

Some of the most ordinary ways for homogeneity magnetic field creation is using of Helmholtz coil. There are a lot of works devoted to the calculation of Helmholtz coils magnetic field [4-8]. One of the others way for uniform magnetic field generation is to use spheroidal coils [9]. The system with bigger number of coils is used to create magnetic field with higher [10], such as: Improved Helmholtz coils, four and six coils system. In this article we made the comparison of magnetic homogeneity in axial direction for four types of coils with similar radius.

${ }^{*}$ Corresponding author: $\underline{\text { iaz5@,tpu.ru }}$ 


\section{Finite element analysis}

Authors used the program Comsol to conduct the finite element analysis. Every coil system was provided with its own task. The calculation was carried in module "Magnetic field". For the windings parameters setting the authors used Multi-turn coil section. The following parameters were set: the number of windings, the current in a conductor and the movement direction. The last parameter was set through the reference edge. It should be noted that the current movement direction in both coils match. The block with manually setting mesh was added to the model in order to receive precise measuring data avoiding fluctuation. It helped to receive data with interval lesser than $1 \mathrm{~mm}$. The stationary type of the task solution was chosen for each of the models as well as iterative solver.

The calculated coils parameters values are persented in the table 1 .

Table 1. Coils parameters.

\begin{tabular}{|c|c|c|}
\hline \multirow{2}{*}{$\mathrm{i}$} & \multicolumn{2}{|c|}{ Parameter value } \\
\hline & $\mathrm{h}, \mathrm{m}$ & $\mathrm{N}$ \\
\hline \multicolumn{3}{|c|}{ Helmholtz coils } \\
\hline- & 0.025 & 10 \\
\hline \multicolumn{3}{|c|}{ Improved Helmholtz coils } \\
\hline Central position & 0 & 5 \\
\hline Edge position & 0.037 & 10 \\
\hline \multicolumn{3}{|c|}{4 coils system } \\
\hline 1 & 0.01 & 10 \\
\hline 2 & 0.045 & 26 \\
\hline \multicolumn{3}{|c|}{6 coils system } \\
\hline 1 & 0.009 & 10 \\
\hline 2 & 0.029 & 12 \\
\hline 3 & 0.0615 & 31 \\
\hline \multicolumn{3}{|c|}{8 coils system } \\
\hline 1 & 0.0135 & 10 \\
\hline 2 & 0.0425 & 12 \\
\hline 3 & 0.0805 & 17 \\
\hline 4 & 0.146 & 34 \\
\hline
\end{tabular}

\section{Calculation results}

The Figure 1-a presents results of the magnetic field calculation of the six coils system in the axial direction with the DC value of $1 \mathrm{~mA}$. The figure 1-b shows the heterogeneity for four coil system in two-dimensional space. The maximum value of the magnetic flux density heterogeneity is observed near coils surface. 


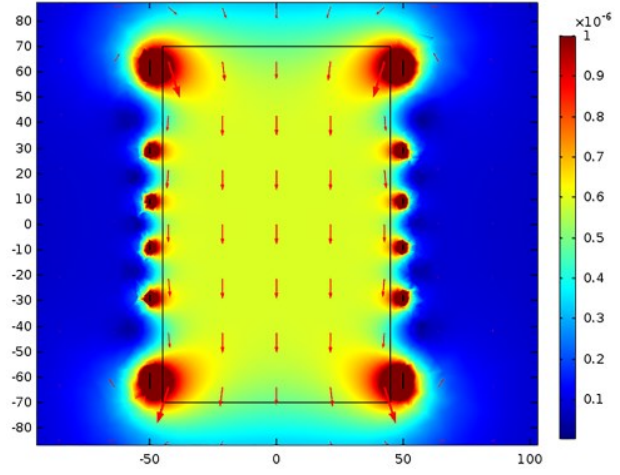

a

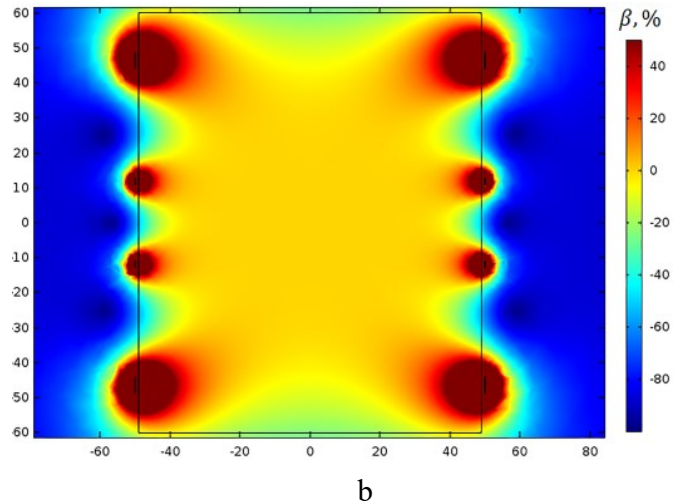

$\mathrm{b}$

Fig. 1. a - the distribution of six coil system magnetic field, b - The heterogeneity of the magnetic field for the four coil system.

The figure 2 presents the heterogeneity of the magnetic field in the axial direction for the considered types of coil systems.

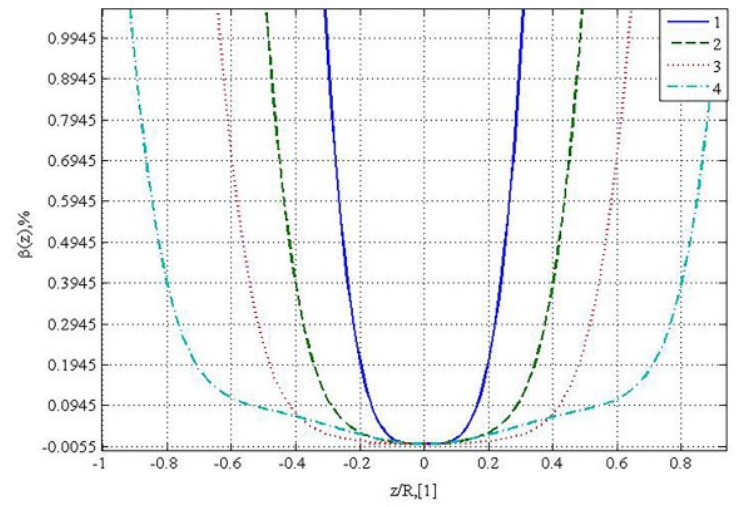

Fig. 2. The heterogeneity of the magnetic field in the axial direction, where $1-$ the Helmholtz coil, 2 - Improved Helmholtz coil, 3 - four coils system, 4 - six coils system.

The changing of one of the parameters presented in the table 1 leads to the magnetic field inhomogeneity change.

The figure 3 shows that the variation of the magnetic field inhomogeneity parameter in the case of the fourth coils pairs depends on the value of ' $h$ '.

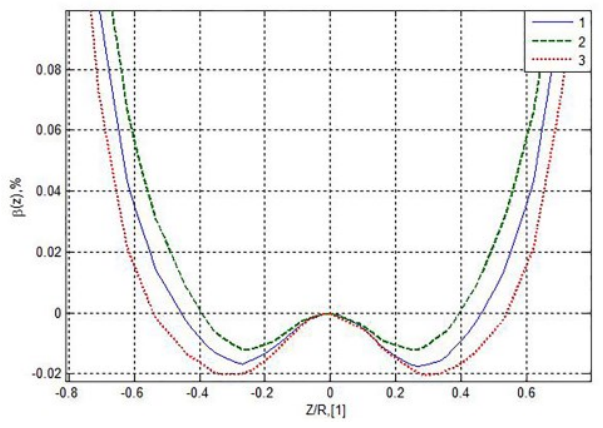

Fig. 3. The heterogeneity of the magnetic field for the 8 coils system in axial direction, where $1-h_{4}=$ $146 \mathrm{~mm} ; 2-h_{4}=145.5 \mathrm{~mm} ; 3-h_{4}=146.5$ 
This figure demonstrates that a slight change in the parameters leads to increase or decrease of the field inhomogeneity. Increasing of the ' $h$ ' for the fourth pair coils allows to expand the area of the desired inhomogeneity.

\section{Conclusion}

The conducted comparison showed the needed heterogeneity of the magnetic field less than $0.1 \%$ is provided by the four and six coils system. The increase of the coils number allows to enlarge the homogeneity of the magnetic field in the axial direction. Thus, four and six coils systems could be used to create a homogeneous magnetic field.

The research is funded from Russian Science Foundation (RSF), Grant Number 17-79-10083.

\section{References}

1. B. Ando, S. Baglio, A.R. Bulsara, C. Trigona, Sen. \&Act. Phys.151,145 (2009)

2. T. Charubin, M. Nowicki, R. Szewczyk, Mechatron 2017 644,613 (2017)

3. A. Tejera,C.Carretero, J.T. Boys, IEEE Trans. Pow. Electron 32(11), 8349 (2016)

4. J. Wang, S. She, S. Zhang, Rev. of Scen. Instr.75, 2175 (2002)

5. V. Baranova, P.Baranov, Mech.\&Mash.(Dyn.),1-4 (2014)

6. S. Magdaleno-Adame, JC. Olivares-Galvan, E. Campero-Littlewood, R. Escarela Perez, E. Blanco-Brisset, In Excerpt from the proceedings of the COMSOL conference (Comsol, Massachusetts, 2010)

7. R. Beiravand. IEEE Trans. Ind. Electron. 64,7120 (2017)

8. M. Hosoya and E. Goto, Rev. Sci. Instrum. 62(10), 2472 (1991)

9. Y. Öztürk, B. Aktaş, Rev. Scien. Instr. 87, 106103 (2016)

10. V. Ogay, Mater. Sci. Eng. 6, 1 (2014)

11. D. Jiles, Introduction to Magnetism and Magnetic Materials, Third Edition (CRC Press, 2015) 